\title{
Laparoscopic Completion of Previous Open Subtotal Cholecystectomy
}

\author{
Symeonidis Dimitrios, MD, PhD, Kelemouridou Efthimia, MD, Koukoulis Georgios, MD, \\ Kapsoritakis Andreas, MD, PhD, Tzovaras George, MD, PhD \\ Department of Surgery, University Hospital of Larissa, Biopolis, Larissa, Greece (Symeonidis D, Kelemouridou E, \\ Koukoulis G, Tzovaras G). \\ Department of Gatroenterology, University Hospital of Larissa, Biopolis, Larissa, Greece (Kapsoritakis A).
}

\begin{abstract}
Introduction: The purpose of this study was to present a challenging case of laparoscopic completion cholecystectomy after a previous open subtotal cholecystectomy. A review of the relevant literature was conducted as well.

Case Description: A 64-year-old patient with a history of open cholecystectomy due to acute cholecystitis presented with right-upper-quadrant abdominal pain, fever, and deranged liver function tests. Magnetic resonance cholangiopancreatography revealed a gallbladder remnant with residual stones and a dilated common bile duct. The patient was submitted to an endoscopic retrograde cholangiopancreatography with sphincterotomy and common bile duct clearance. As symptoms persisted, the completion of the previous subtotal cholecystectomy was decided. A laparoscopic completion cholecystectomy was attempted and was completed successfully.
\end{abstract}

Discussion: Up to 10 cases of this certain sequence have been reported to date. Attempts for the laparoscopic removal of the gallbladder remnant should not be averted based on the history of open subtotal cholecystectomy.

Key Words: Completion cholecystectomy, Recurrent gallstones, Subtotal cholecystectomy.

Citation Dimitrios S, Efthimia K, Georgios K, Andreas K, George T. Laparoscopic completion of previous open subtotal cholecystectomy. CRSLS e2014.00251. DOI 10.4293/CRSLS.2014.00251.

Copyright (C) 2014 SLS This is an open-access article distributed under the terms of the Creative Commons Attribution-Noncommercial-ShareAlike 3.0 Unported license, which permits unrestricted noncommercial use, distribution, and reproduction in any medium, provided the original author and source are credited.

Address correspondence to: Dimitrios Symeonidis, 24 Collier Place, EH16 4PZ, Edinburgh, UK. Telephone: +4407450929994, E-mail: simeonid@hotmail.com

\section{INTRODUCTION}

Subtotal cholecystectomy has been traditionally considered as the last resort in cases of difficult cholecystectomies to reduce the incidence of an inadvertent bile duct or arterial injury. ${ }^{1}$ The fear of complications arising from the retained gallbladder stump caused skepticism regarding the actual utility of the approach. However, balancing the risks between an iatrogenic bile duct and/or arterial injury and that of the stump-related complications, subtotal cholecystectomy, when appropriate, appears a decision of wisdom. ${ }^{1}$ This is the case where the extensive gallbladder inflammation renders an accurate identification of the anatomy of the extrahepatic biliary tree problematic. ${ }^{1}$

Leaving a gallbladder remnant in situ during cholecystectomy is either a purposeful or an involuntary incident from the surgeon's viewpoint. Failure to properly identify the parts of the gallbladder intraoperatively is mainly responsible for the latter. ${ }^{2}$ Besides the increased incidence of immediate postoperative complications such a biliary leak, the longterm morbidity is not significant when a part of the gallbladder remains in continuity with the biliary tree. ${ }^{2}$ Symptoms, when present, are associated with the presence of gallstones and include right upper-quadrant pain, positive Murphy sign, and jaundice. ${ }^{2}$ The diagnosis of a symptomatic gallbladder remnant can be especially challenging. Abdominal ultrasound, magnetic resonance cholangiopancreatography (MRCP), and endoscopic retrograde cholangiopancreatography (ERCP) have all been used to document the diagnosis and mainly to correlate the presence of a gallbladder remnant with the patient's symptoms. ${ }^{2}$

The purpose of the present study was to present an interesting case where the removal of a symptomatic gallbladder stump laparoscopically proved feasible after a 
previous open subtotal cholecystectomy performed elsewhere several years ago. A review of the existing relevant literature was conducted as well.

\section{MATERIALS AND METHODS}

A 64-year-old female patient was admitted in the emergency department of the University Hospital of Larissa complaining of upper-right-quadrant abdominal pain and fever over the last few hours. Past medical history included an open cholecystectomy performed elsewhere 6 years earlier due to acute cholecystitis. A couple of episodes of epigastric pain and/or bloating during the past 12 months that, however, did not require admission to the hospital, were elicited from patient's history.

Physical examination revealed a relatively tender abdomen and a positive Murphy sign. The patient had a body temperature of $38.5^{\circ} \mathrm{C}$, blood pressure of $110 / 70 \mathrm{~mm} \mathrm{Hg}$, and heart rate at 89 beats per minute. Laboratory workup revealed deranged liver function tests (serum glutamate oxaloacetate transaminase: 189, alkaline phosphatase: 340, gamma-glutamyl transferase: 400) and a serum bilirubin level of $4.1 \mathrm{~g} / \mathrm{dL}$. As abdominal ultrasound findings were equivocal, the patient was subsequently investigated with an MRCP that revealed a gallbladder remnant with residual stones and common bile duct (CBD) dilatation (Figures 1 and 2). Having the diagnostic criteria of acute cholangitis fulfilled, antibiotics were commenced and the patient was submitted to an ERCP with sphincterotomy and CBD clearance. Due to the persistence of right-upperquadrant pain even several days after the ERCP and given that post-ERCP complications-mainly pancreatitis-were primarily excluded, the option of completing a previous, apparently, subtotal cholecystectomy was adopted.

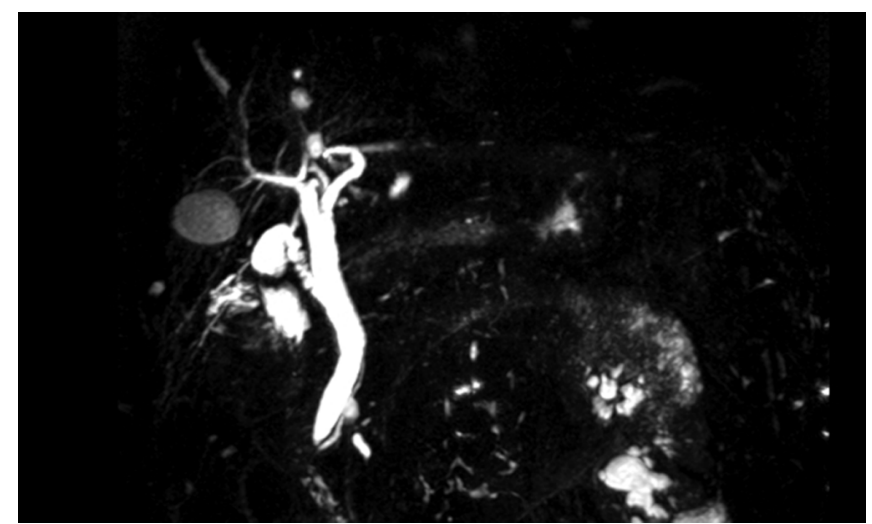

Figure 1. Magnetic retrograde cholangiogram image showing the gallbladder stump in continuity with the extrahepatic biliary tree.

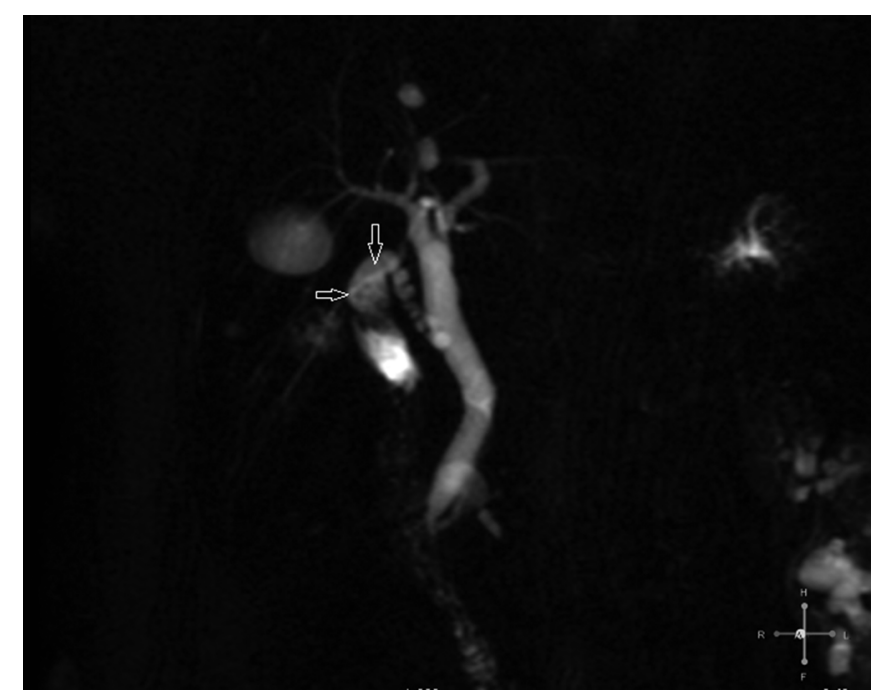

Figure 2. Magnetic retrograde cholangiogram image showing the filling defects within the gallbladder stump and the cystic duct (white arrows) as well as the dilated CBD

\section{RESULTS}

Although the traditional open approach via a Kocher incision was followed during the first procedure, we attempted the laparoscopic approach for the completion of the cholecystectomy. Regarding the procedure itself, an umbilical 10-mm optical trocar was inserted using the open Hasson technique, a pneumoperitoneum was set at $12 \mathrm{~mm} \mathrm{Hg}$ and laparoscopy was initiated. The subhepatic area was covered with relatively dense adhesions not, however, precluding a safe laparoscopic dissection. Then, another 10-mm trocar just below the xiphoid and $25-\mathrm{mm}$ trocars at the routine positioning were inserted for the necessary instrumentation.

After having the adhesions, as well as the hepatic flexure dissected off the liver bed using a combination of sharp and blunt dissection as appropriate, with the use of as minimal as possible diathermy, we were able to identify the gallbladder remnant that was bluntly dissected free from the duodenum. The cystic duct-CBD confluence was identified while the gallbladder remnant was completely freed from its adhesive attachments. Having identified the pertinent anatomical structures, the cystic duct was divided between clips close to its confluence to the CBD. One silicone drain was finally left in place.

The patient had an uneventful recovery and was discharged from the hospital on postoperative day 2 . 


\section{DISCUSSION}

Over the past 2 decades, we have witnessed a gradual modification of the indications of laparoscopic cholecystectomy for acute cholecystitis. Based on the high incidence of bile and/or arterial injuries, acute cholecystitis had been initially considered as an absolute or relative contraindication for the laparoscopic approach. ${ }^{3}$ Indeed, acute inflammation distorts the anatomy due to the edema and the formation of dense adhesions increasing significantly the risk of operative complications.,3 Within this context, many patients were denied the benefits of laparoscopic surgery for fear of complications. With the widespread use of laparoscopy and the experience gained, "early" laparoscopic cholecystectomy has evolved into the standard of care in acute cholecystitis. ${ }^{4,5}$ Indeed, several studies reported on laparoscopic cholecystectomy in the setting of acute cholecystitis and confirmed the safety of this approach. ${ }^{6-8}$

However, regardless of the approach-open or laparoscopic - the removal of the acutely inflamed gallbladder is a procedure of a significantly higher level of difficulty compared with an elective removal of a "normal" gallbladder. ${ }^{1,9}$ Even conventional laparotomy cannot sometimes guarantee a straightforward, complication-free cholecystectomy. Dissection around the Callot triangle on the background of inflammation can be especially challenging, increasing the risk of bile and/or arterial injuries. ${ }^{10-12}$ Certainly, achieving a technically safe complete excision of the gallbladder is always the desired goal. However, in case of difficulties, the partial removal of the gallbladder wall, that is, subtotal cholecystectomy has been proposed as a reasonable alternative option that can sufficiently address the patient's symptoms without risking an inadvertent iatrogenic injury. The approach is even advocated during laparoscopic cholecystectomy in an attempt to avoid the conversion to laparotomy. ${ }^{13,14}$ Proactively thinking, the use of Percutaneous cholecystostomy in severe acute cholecystitis can prove especially important. 5 Draining externally, an acutely inflamed gallbladder can (1) relieve these septic patients of their symptoms, (2) provide the necessary time for the inflammation to settle down, and (3) substantially increase the chances of achieving a complete removal of the gallbladder when definite treatment is indicated, that is, cholecystectomy. ${ }^{5}$

Unfortunately, by definition there are no prospective randomized studies comparing total with subtotal cholecystectomy for acute cholecystitis. The "doing-less-harm" concept of subtotal cholecystectomy could not be stan- dardized within the context of a prospective randomization, perpetuating the global debate regarding the actual utility of the approach. However, the fact that subtotal cholecystectomy has a non-negligible role in the surgical armamentarium for the management of severe acute cholecystitis is adequately underscored from the results of the available retrospective cohort studies. ${ }^{13,14}$ Nevertheless, evaluating the reported short-term results is mainly problematic as statistical analysis between the compared groups - total and subtotal-is not feasible. However, what seems of most importance is the assessment of the long-term results of an approach that leaves a part of the gallbladder, almost invariably the Hartmann pouch, in situ. Studies do not seem to attach a significantly higher incidence of long-term complications such as recurrent or residual gallstone disease related to the retained gallbladder remnant. ${ }^{2,13,14}$

Despite the fact that the incidence of complications following subtotal cholecystectomy is not high enough to suggest the inappropriateness of this particular approach, complications do supervene. Causes include bile duct residual stones or strictures, and, rarely, stone recurrence in the gallbladder remnant. ${ }^{14}$ Whereas pathologies associated with the $\mathrm{CBD}$ require at least initially endoscopic intervention, the recurrence of stones within the gallbladder remnant flanked with relevant symptoms requires surgical treatment. ${ }^{14,15}$ Although the laparoscopic approach has been used for the completion of an initial laparoscopic subtotal cholecystectomy, the usual clinical scenario consists of an open completion cholecystectomy, that is, removal of the retained gallbladder stump regardless of the initial approach. ${ }^{15,16}$

In the present report, a reverse sequence was used: a laparoscopic completion cholecystectomy was performed after an initial open subtotal cholecystectomy. To our knowledge, a total of 10 cases of this challenging sequence-laparoscopic after open-have been reported in the literature. ${ }^{17,18}$ The rationale for performing an open subtotal cholecystectomy in the first place, that is., severe inflammation with difficulties in identifying the anatomy, stands as an argument as well to avert an "ambitious" attempt for the removal of the retained gallbladder using the laparoscopic approach in the symptomatic patient.

We certainly do not intend to reach a solid conclusion based on the successful operative result of a single case; however, what the present report and the previously published series have proved is that such attempts are, at least, not futile. In addition, the adequate expertise in the lapa- 
roscopic surgery could incisively influence the outcome of such attempts. Data in the literature regarding failures could not possibly exist; however, we would consider the competence in laparoscopic cholecystectomy in cases of acute inflammation as an adequate, although nonvalidated, proof of proficiency for such attempts. Having in mind the well-established advantages of laparoscopic surgery over conventional open surgery, ${ }^{1,4,5}$ attempting a laparoscopic completion cholecystectomy even after an open subtotal cholecystectomy appears a reasonable strategy in terms of patient's benefits, provided this can be performed without risking an iatrogenic injury. Generalizing any suggestion as such is not possible on the basis of case reports and every particular case should be managed individually. It seems, however, that laparoscopy under these circumstances represents a reasonable first step that can provide the required information for selecting the optimal final approach, that is, either proceeding with laparoscopy or converting to an open procedure.

\section{CONCLUSIONS}

The laparoscopic removal of a retained gallbladder stump after open subtotal cholecystectomy proved feasible in the given patient. Attempts for laparoscopic removal of the gallbladder remnant should not be averted based only on the history of an open subtotal cholecystectomy.

\section{Consent}

Written informed consent was obtained from the patient for publication of this case report and any accompanying images. A copy of the written consent is available for review by the Editor-in-Chief of this journal.

\section{References:}

1. Borzellino G, Sauerland S, Minicozzi AM, et al. Laparoscopic cholecystectomy for severe acute cholecystitis: a meta-analysis of results. Surg Endosc. 2008;22(1):8-15.

2. Beldi G, Glättli A. Laparoscopic subtotal cholecystectomy for severe cholecystitis. Surg Endosc. 2003;17(9):1437-1439.

3. Hermann RE. Surgery for acute and chronic cholecystitis. Surg Clin North Am. 1990;70(6):1263-1275.

4. Perissat J. Laparoscopic cholecystectomy: the European experience. Am J Surg. 1993;165(4):444-449.
5. Mayumi T, Takada T, Kawarada Y, et al. Results of the Tokyo Consensus Meeting Tokyo Guidelines. J Hepatobiliary Pancreat Surg. 2007;14(1):114-121.

6. Kum CK, Goh PM, Isaac JR, Tekant Y, Ngoi SS. Laparoscopic cholecystectomy for acute cholecystitis. Br J Surg. 1994;81(11): 1651-1654.

7. Fabre JM, Fagot H, Domergue J, et al. Laparoscopic cholecystectomy in complicated cholelithiasis. Surg Endosc. 1994; 8(10):1198-1201.

8. Singer JA, McKeen RV. Laparoscopic cholecystectomy for acute or gangrenous cholecystitis. Am Surg. 1994;60(5):326-328.

9. Bakr AA, Khalil ME, Esmat GE. Acute cholecystitis is an indication for laparoscopic cholecystectomy: a prospective study. JSLS. 1997;1(2):119-123.

10. Davis B, Castaneda G, Lopez J. Subtotal cholecystectomy versus total cholecystectomy in complicated cholecystitis. Am Surg. 2012;78(7):814-817.

11. Bornman PC, Terblanche J. Subtotal cholecystectomy: for the difficult gallbladder in portal hypertension and cholecystitis. Surgery. 1985;98(1):1-6.

12. Cottier DJ, McKay C, Anderson JR. Subtotal cholecystectomy. Br J Surg. 1991;78(11):1326-1328.

13. Nakajima J, Sasaki A, Obuchi T, Baba S, Nitta H, Wakabayashi G. Laparoscopic subtotal cholecystectomy for severe cholecystitis. Surg Today. 2009;39(10):870-875.

14. Henneman D, da Costa DW, Vrouenraets BC, van Wagensveld BA, Lagarde SM. Laparoscopic partial cholecystectomy for the difficult gallbladder: a systematic review. Surg Endosc. 2013; 27(2):351-358.

15. Demetriades H, Pramateftakis MG, Kanellos I, Angelopoulos S, Mantzoros I, Betsis D. Retained gallbladder remnant after laparoscopic cholecystectomy. J Laparoendosc Adv Surg Tech A. 2008;18(2):276-279.

16. Pernice LM, Andreoli F. Laparoscopic treatment of stone recurrence in a gallbladder remnant: report of an additional case and literature review. J Gastrointest Surg. 2009;13(11):20842091.

17. Chowbey P, Soni V, Sharma A, Khullar R, Baijal M. Residual gallstone disease: laparoscopic management. Indian J Surg. 2010;72(3):220-225

18. Gurel M, Sare M, Gurer S, Hilmioglu F. Laparoscopic removal of a gallbladder remnant. Surg Laparosc Endosc. 1995; 5(5):410-411. 